\title{
Structure and mechanical properties of high-weight-bearing and low-weight- bearing areas of hip cartilage at the micro- and nano-levels
}

\author{
Jiang-Bo Guo ${ }^{1,2+}$, Ting Liang ${ }^{2 \dagger}$, Yan-Jun Che ${ }^{1,2+}$, Hui-Lin Yang ${ }^{1,2}$ and Zong-Ping Luo ${ }^{2^{*}}$ (D)
}

\begin{abstract}
Background: Articular cartilage has a high-weight-bearing area and a low-weight-bearing area, the macroscopic elastic moduli of the two regions are different. Chondrocytes are affected by the applied force at the microscopic level. Currently, the modulus of the two areas at the micro and nano levels is unknown, and studies on the relationship between macro-, micro- and nano-scale elastic moduli are limited. Such information may be important for further understanding of cartilage mechanics. Moreover, the surface morphology, proteoglycan content, and micro and nano structure of the two areas, which influences the mechanical properties of cartilage should be discussed.

Methods: Safranin-O/Fast Green staining was used to evaluate the surface morphology and semi-quantify proteoglycan content of porcine femoral head cartilage between the two weight-bearing areas. The unconfined compression test was used to determine the macro elastic modulus. Atomic force microscope was used to measure the micro and nano compressive elastic modulus as well as the nano structure. Scanning electron microscope was employed to evaluate the micro structure.

Results: No significant differences in the fibrillation index were observed between two areas $(P=0.5512)$. The Safranin-O index of the high-weight-bearing area was significantly higher than that of the low-weight-bearing area $(P=0.0387)$. The compressive elastic modulus of the high-weight-bearing area at the macro and micro level was significantly higher than that of the low-weight-bearing area $(P=0.0411$ for macro-scale, and $P=0.0001$ for microscale), while no statistically significant differences were observed in the elastic modulus of collagen fibrils at the nano level $(P=0.8544)$. The density of the collagen fibers was significantly lower in the high-weight-bearing area $(P=0.0177)$. No significant differences were observed in the structure and diameter of the collagen fibers between the two areas $(P=0.7361)$.

(Continued on next page)
\end{abstract}

\footnotetext{
* Correspondence: zongping_luo@yahoo.com

†Jiang-Bo Guo, Ting Liang and Yan-Jun Che contributed equally to this work.

${ }^{2}$ Department of Orthopaedics, Orthopaedic Institute, the First Affiliated

Hospital, Soochow University, Suzhou, Jiangsu 215006, People's Republic of

China

Full list of author information is available at the end of the article
}

(c) The Author(s). 2020 Open Access This article is licensed under a Creative Commons Attribution 4.0 International License, which permits use, sharing, adaptation, distribution and reproduction in any medium or format, as long as you give appropriate credit to the original author(s) and the source, provide a link to the Creative Commons licence, and indicate if changes were made. The images or other third party material in this article are included in the article's Creative Commons licence, unless indicated otherwise in a credit line to the material. If material is not included in the article's Creative Commons licence and your intended use is not permitted by statutory regulation or exceeds the permitted use, you will need to obtain permission directly from the copyright holder. To view a copy of this licence, visit http://creativecommons.org/licenses/by/4.0/ The Creative Commons Public Domain Dedication waiver (http://creativecommons.org/publicdomain/zero/1.0/) applies to the data made available in this article, unless otherwise stated in a credit line to the data. 


\begin{abstract}
(Continued from previous page)
Conclusions: A higher proteoglycan content correlated with a higher compressive elastic modulus of the highweight-bearing area at the micro level than that of the low-weight-bearing area, which was consistent with the trend observed from the macroscopic compressive elastic modulus. The weight-bearing level was not associated with the elastic modulus of individual collagen fibers and the diameter at the nano level. The micro structure of cartilage may influence the macro- and micro-scale elastic modulus.
\end{abstract}

Keywords: Cartilage, Collagen fiber, Elastic modulus, Micro-scale, Nano-scale, Weight-bearing

\section{Background}

Articular cartilage is an important weight-bearing structure of the human body. In particular, the hip joint is the comparatively large weight-bearing joint that plays an important role in joint lubrication, joint friction reduction, and pressure damping [1]. Articular cartilage consists of a small number of chondrocytes and a large number of extracellular matrices. Chondrocytes maintain the normal metabolism of the cartilage. The extracellular matrix mainly contains type II collagen, proteoglycans, and water, and the extracellular matrix content and the cross-linking mechanism of collagen affect normal functioning of cartilage [2]. Articular cartilage tissue is subject to long-term cyclic loads, and cartilage is closely related to the load it carries. For example, osteoarthritis is closely related to stress, since long-term exposure to excessive stress can cause osteoarthritis. An important method in the conservative treatment of osteoarthritis is to relieve the weight load of the joint [3].

Regarding articular cartilage, weight-bearing is nonuniform and spatially specific. Based on the different cartilage locations, the femoral head cartilage area can be easily divided into a high-weight-bearing area (HWA) and a low-weight-bearing area (LWA) $[4,5]$. The relationship between HWA and LWA is very close. For example, cartilage with HWA subjected to long-term cyclic stress is prone to develop degenerative damages, such as osteoarthritis, and transplantation of autologous LWA cartilage at the damaged HWA has been used in the clinic to repair joint injury [6-8]. Regarding the design of tissue material for cartilage repair, it is necessary to consider the magnitude of cartilage load under normal conditions, as well as the difference in mechanical properties between different weight-bearing areas. Both parameters are critical for the design of future cartilage substitute materials $[9,10]$. Macro-mechanical studies have shown that different loading areas of cartilage have different mechanical properties [11-15], but the chondrocytes are affected at the microscopic level by the applied force [16]. From a microscopic point of view, the chondrocytes are very sensitive to microscopic forces and the surrounding micro-nano structures, and mechanical properties alter with the load changes in the surrounding microscopic environment [17-19]. Therefore, the mechanical properties of collagen fibers in the HWA and the LWA at the micro-nano level, and the relationship between macro and micro-nano levels are of utmost importance for further understanding of the cartilage mechanical properties. Moreover, the mechanical properties of cartilage are mainly related to the crosslinking mechanism of collagen fibers and proteoglycan content [20], and such significant factors need to be discussed.

Given the aforementioned micromechanical properties, and because the micro level is relatively close to the macroscopic level, it was assumed that the elastic modulus at the micro level should be consistent with the macroscopic compressive elastic modulus trend of the cartilage. For nano-scale mechanical properties, individual fibers are measured, therefore, the differences between the two regions may be minor. At the micro level, the micro structure of cartilage in the HWA should be more conducive to load-bearing. However, the differences in structure at the nano-scale are less obvious due to the smaller range of the detection region. The proteoglycan content should be consistent with the trend of elastic modulus between two areas. During physiological function, cartilage is a load-bearing component, and under compression conditions, its mechanical properties are consistent with the physiological conditions of the body. Therefore, an atomic force microscope (AFM) mechanical test was performed to measure the microand nano-scale compressive elastic modulus and the nano-scale structure of cartilage. In addition, scanning electron microscope (SEM) was employed to scan and evaluate the structure of micron-sized cartilage. Safranin-O/Fast Green staining was performed to evaluate the surface morphology and to semi-quantify proteoglycan content of the cartilage.

\section{Materials and methods}

\section{Sample preparation}

In this study, healthy femoral head cartilage from 8month-old porcine $(n=6)$ without degeneration was used. Fresh healthy porcine femoral heads that were slaughtered the day before, were purchased from the same local malls (Yangcanli Market, Jiangsu Province, 
China) in the morning. No significant differences in the weight and volume of the porcine femoral heads were observed. To expose the femoral head cartilage, muscles and ligaments were sheared and cleaned. The weightbearing condition of the cartilage of the femoral head of porcine is similar to that of humans, and two different weight-bearing zones were marked (Fig. 1a), including the HWA above the femoral head, and the LWA below the femoral head $[4,5]$. The entire cartilage layer was harvested using an electric drill connected with a broken nail extractor (diameter $=8 \mathrm{~mm}$ ), then divided into four specimens using surgical blades for subsequent experiments, each specimen corresponded to a specific experiment (Fig. 1b). Specimens for histological analysis were fixed in 10\% neutral buffered formalin (Shanghai Yuanye Bio-Technology Co Ltd., Shanghai, China) for $24 \mathrm{~h}$, whereas other specimens stored in a refrigerator at $20^{\circ} \mathrm{C}$ for later use [21]. All animal experiments were strictly performed under the guidelines of the Chinese Council for Animal Care, approved by the Animal Care Committee of the Laboratory Animal at School of Medicine, SooChow University.

\section{Histological analysis}

HWA and LWA specimens were fixed in 10\% neutral buffered formalin (Shanghai Yuanye Bio-Technology Co Ltd., Shanghai, China) for $24 \mathrm{~h}$. After gradient alcohol (Sinopharm Chemical Reagent Co Ltd., Shanghai, China) dehydration, specimens were paraffin-embedded (Leica, Richmond, VA, USA) for serial sectioning using a histotome (Leica, Richmond, VA, USA) at a thickness of $6 \mu \mathrm{m}$. Safranin O/Fast Green staining was performed on the cartilage tissue sections according to the kit instructions (Beijing Solarbio Science \& Technology Co Ltd., Beijing, China). Staining was evaluated using a binocular microscope (XSP-2CA, Shanghai, China). Two histologic sections of each sample were used to obtain the fibrillation index (FI) to determine the cartilage surface morphology at a magnification of $100 \times$ as previously described [22]. Per sample, two sections were used for the Safranin-O index to semi-quantify the cartilage proteoglycan content in the top $20 \mu \mathrm{m}$ at a magnification of $200 \times$ as previously described [23].

\section{Macro elastic modulus of cartilage obtained by the unconfined compression test}

Within $24 \mathrm{~h}$ after specimen removal, an unconfined compression test to $60 \%$ strain was performed at a displacement rate of $0.6 \mathrm{~mm} / \mathrm{min}$ using a biomechanical testing machine (Shanghai Heng Wing Precision Instrument Co Ltd., Shanghai, China) and a $50 \mathrm{~N}$ mechanical sensor. Each sample ( $n=6$ per area) was tested once. The macro elastic modulus, the slope of the stress strain curve, was calculated using the linear region of the curve in Origin 8.0 (Northampton, MA, USA) [24, 25].

\section{Micro-nano elastic modulus measurement and nano-sized collagen fiber morphological observation by AFM}

Within $24 \mathrm{~h}$ after specimen removal, the cartilage specimen was embedded in Optimal Cutting Temperature (OCT) compound, and frozen sections were cut to get the top layer of the cartilage (thickness $=20 \mu \mathrm{m}$ ) using a freezing microtome (Leica, Richmond, VA, USA). Sections were adhered to a glass slide, and biomechanical analysis was performed using an AFM scanner (Dimension ICON, Bruker, USA). To obtain the micro-scale elastic modulus, at the micrometer scale, twelve locations per stress zone were randomly pressed using a spherical tip at a diameter of $5 \mu \mathrm{m}$. At the nano scale, fifteen collagen fibrils were randomly selected per area to measure the nano-biomechanical property using a ScanAsyst-Air probe at a radius of $5 \mathrm{~nm}$. The cartilage nano-scale topography was also scanned at the nanometer level. Fifteen collagen fibers were randomly selected per area from cartilage nano images, and the diameter of collagen fibers was determined by using the

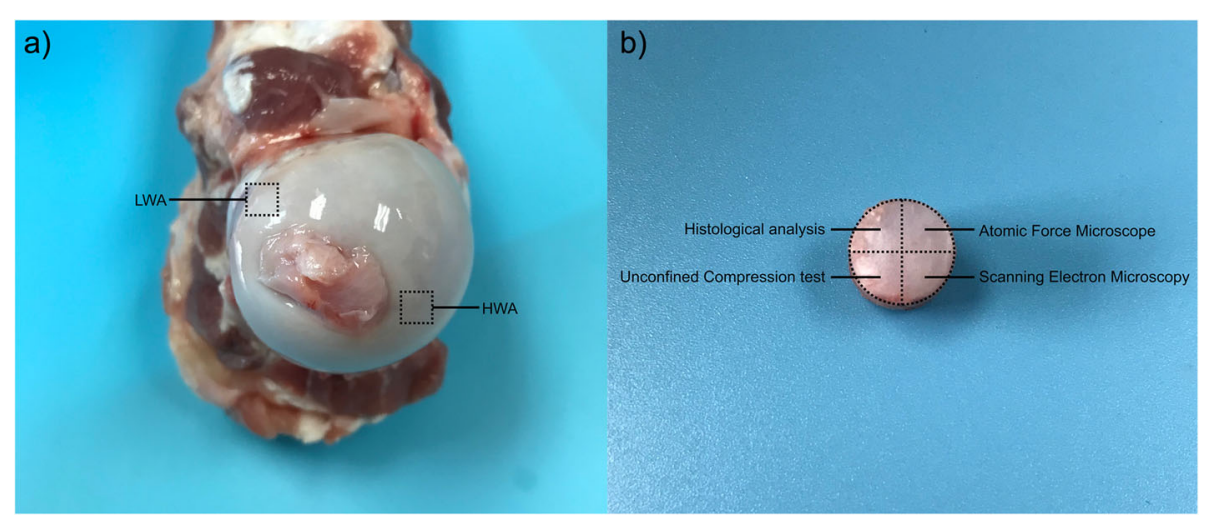

Fig. 1 Sample preparation. (a) High-weight-bearing area (HWA) and Low-weight-bearing area (LWA) of porcine femoral head cartilage (b) Experimental design 
scale tool in Adobe Photoshop CS5 (Adobe Systems Incorporated, San Jose, CA, USA) [26].

\section{Morphological observation of micron-sized collagen fibers by SEM}

The cartilage samples were fixed in $2.5 \%$ glutaraldehyde for $4 \mathrm{~h}$ at $4{ }^{\circ} \mathrm{C}$, then washed in PBS $0.01 \mathrm{M}$ for $2 \mathrm{~h}$. Then, samples were processed by the $\mathrm{NaOH}$ cell-maceration method [27, 28]. In brief, samples were immersed in $10 \% \mathrm{NaOH}$ solution for 3-4 days at room temperature, then rinsed in distilled water for 3 days. Next, samples were immersed in $1 \%$ tannic acid for $2 \mathrm{~h}$. Subsequently, samples were dehydrated with 70, 80, 90, and 100\% alcohol for $4 \mathrm{~h}$, dried in a critical point dryer (Leica, Richmond, VA, USA) with liquid $\mathrm{CO}_{2}$, and coated with ion sputter (Quorum Technologies, East Sussex, UK). The collagen cross-linking mechanism including the direction of collagen fibers alignment, spacing and their differences along the HWA and the LWA were observed by SEM (Quanta 250, FEI, Hillsboro, Oregon, USA) at a magnification of $1000 \times$. The density of collagen fibers was calculated in fifteen regions of each weight-bearing area at a magnification of $3000 \times$.

\section{Statistical analysis}

Data from all experiments are presented as the means \pm standard deviation or median (interquartile range, IQR) as appropriate. Data management and statistical analysis were performed using Microsoft Excel 2016 (Microsoft Corp., Edmond, WA, USA) and GraphPad Prism 8.0.2 (GraphPad Software Inc., San Diego, CA, USA). Significant differences in FI, Safranin-O index, macro-scale elastic modulus, and the density of collagen fiber between two groups were obtained by using the MannWhitney test, and significant differences in the microand nano-scale elastic modulus and the diameter of collagen fibers between two groups were obtained by using the unpaired t-test. Statistical significance was set at $p \leq$ 0.05 .

\section{Results}

\section{Histological analysis}

Representative staining results are presented in Fig. 2. Figure $2 \mathrm{a}$ and $\mathrm{c}$ represented HWA, while $2 \mathrm{~b}$ and $2 \mathrm{~d}$ represented LWA. The median FI of HWA was 98.10 (IQR 97.03-105.67), and the median FI of LWA was 97.13 (IQR 96.74-106.31). No statistically significant differences in FI were observed between the two areas (Fig. 2e, $P=0.5512$ ). The top $20 \mu \mathrm{m}$ of LWA showed Safranin-O staining loss as indicated in Fig. $2 \mathrm{~b}$ and $\mathrm{d}$. The median Safranin-O index of HWA was 164.10 (IQR 138.70-211.02), while the median Safranin-O index of LWA was 116.30 (IQR 102.53-204.70). The Safranin-O index of HWA was significantly higher when compared to that of LWA (Fig. 2f, $P=0.0387$ ).

\section{Femoral head cartilage macro-, micro- and nano-scale elastic modulus}

The median macro-scale elastic modulus of the HWA was 0.32 (IQR $0.27-0.33$ ) MPa, while the median macroscale elastic modulus of the LWA was 0.15 (IQR $0.12-$ 0.22) MPa. Our data showed that the macro-scale elastic modulus of the femoral head cartilage was significantly higher in the HWA when compared to the LWA (Fig. 3a, $P=0.0411)$. The micro-scale elastic modulus of the HWA was $(0.44 \pm 0.07) \mathrm{MPa}$, while the micro-scale elastic modulus of the LWA was $(0.32 \pm 0.06) \mathrm{MPa}$. In addition, the micro-scale elastic modulus of the femoral head cartilage was significantly higher in the HWA when compared to the LWA (Fig. $3 \mathrm{~b}, P=0.0001$ ). The nanoscale elastic modulus of the HWA collagen fibers was $(1.29 \pm 0.20) \mathrm{GPa}$, while the nano-scale elastic modulus of the LWA collagen fibers was $(1.28 \pm 0.15) \mathrm{GPa}$. No statistically significant differences were observed in the elastic modulus of collagen fibril at nano-scale between the two regions (Fig. 3c, $P=0.8544$ ).

\section{Femoral head cartilage nanostructure}

A representative image of the nanostructure of cartilage is presented in the Fig. 4. At the nano-scale, the arrangement and alignment of collagen fibers in the two weight-bearing areas tended to be randomly distributed. There are no specific rules to follow. The diameter of cartilage collagen fibers in the HWA was $(98.73 \pm 19.50)$ $\mathrm{nm}$, while the diameter of collagen fibers in the LWA was $(96.21 \pm 20.91) \mathrm{nm}$. No statistically significant differences were observed in the diameter of collagen fibrils at the nano-scale level between the two regions (Fig. 4c, $P=0.7361$ ). Furthermore, no significant differences were observed between the nanoscale morphology of the cartilage surface of the HWA and the LWA on the AFM scans.

\section{Femoral head cartilage microstructure}

The SEM results at $1000 \mathrm{x}$ magnification (Fig. 5a and b) showed that, although the collagen fiber network tended to be randomly arranged in the two weight-bearing areas, both areas have some collagen fibers arranged in a radial direction. The arrangement of collagen fibers and collagen fibers in the HWA is more organized and regular when compared to that in the LWA (Fig. 5a). In the LWA, several collagen fibers tended to aggregate, and spacing of collagen fibers in the LWA was not as well arranged when compared to that in the HWA (Fig. 5b). Calculation of the density of collagen fibers at 3000x magnification (Fig. 5c and d) showed that the median density of collagen fibers in the HWA was 50,000.00 

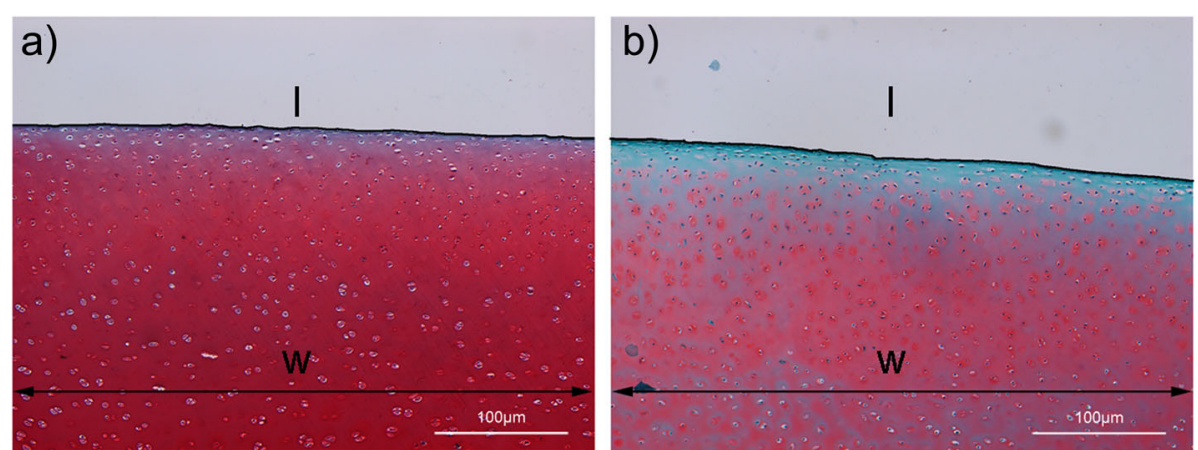

c)

d)
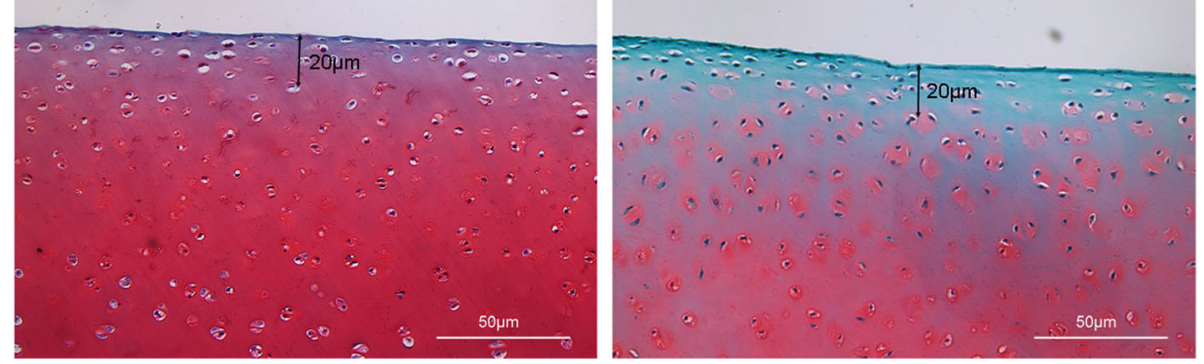

e) $(\mathrm{I} / \mathrm{w}) \times 100$

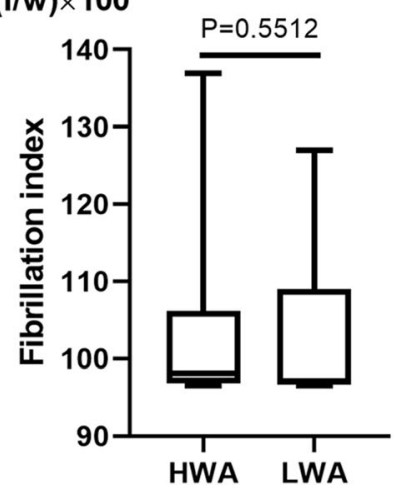

f)

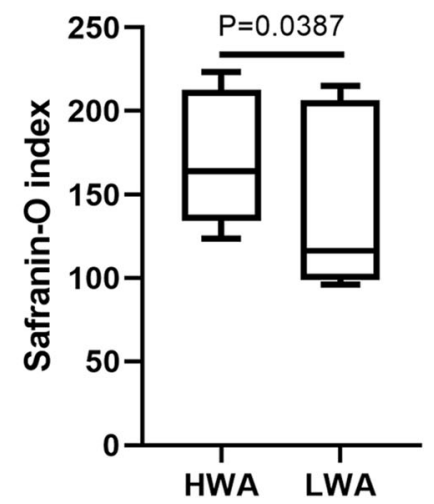

Fig. 2 Representative Safranin-O staining images of two areas. (a) High-weight-bearing area (HWA) at a magnification of $100 \times$. (b) Low-weightbearing area (LWA) at a magnification of $100 \times$. (c) HWA at a magnification of $200 \times$. (d) LWA at a magnification of $200 \times$. (e) Fibrillation index (Fl) results of two areas. (f). Safranin-O index results of two areas ( $n=6$ per area)
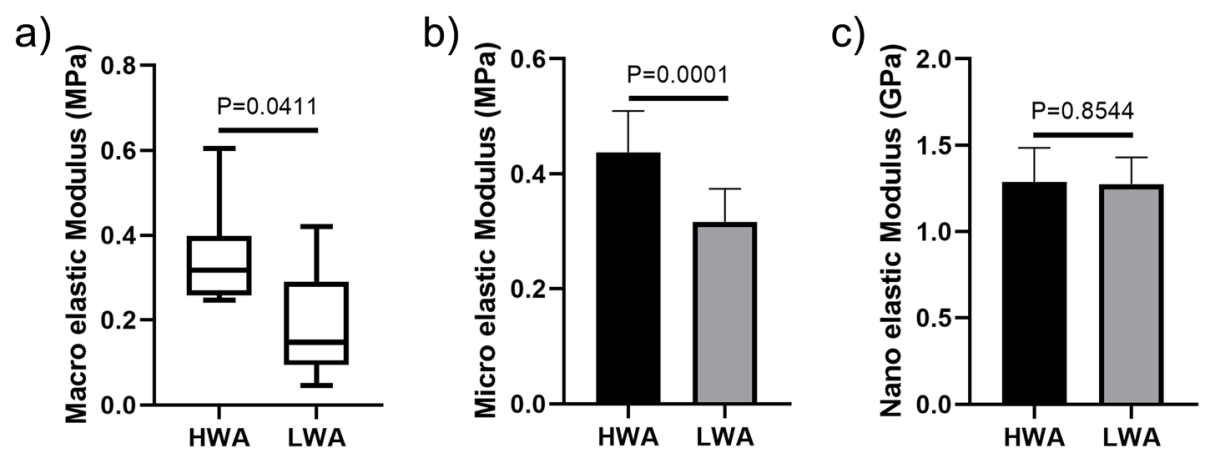

Fig. 3 Macro-, micro- and nano-scale elastic modulus of high-weight-bearing area (HWA) and low-weight-bearing area (LWA). (a) Macro-scale elastic modulus results. (b) Micro-scale elastic modulus results. (c) Nano-scale elastic modulus results ( $n=6$ per area) 

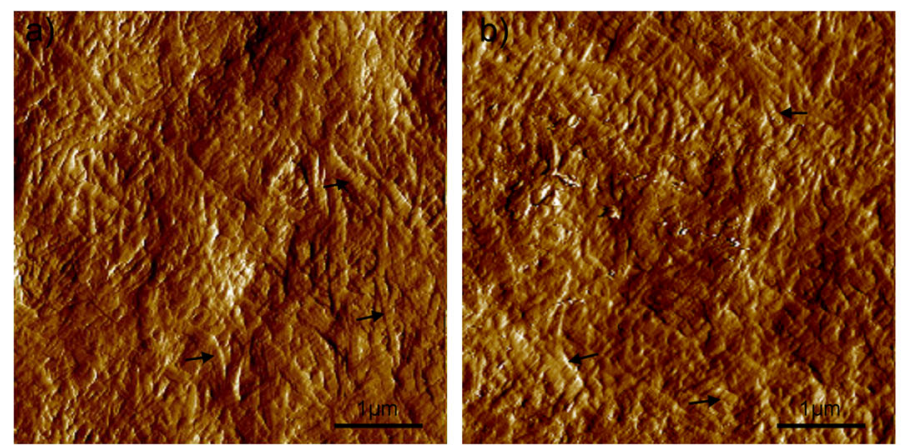

c)

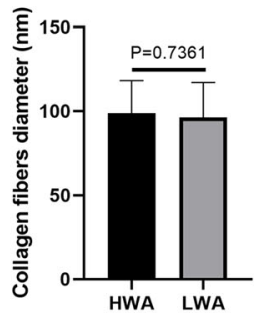

Fig. 4 Representative image of the atomic force microscope (AFM) nano-scale morphology of cartilage of two areas. (a) High-weight-bearing area (HWA) nano-scale cartilage morphology. (b) Low-weight-bearing area (LWA) nano-scale cartilage morphology. (c) Diameter of collagen fibers of two areas ( $n=6$ per area)

(IQR 50000.00-55,000.00)/ $\mathrm{mm}^{2}$, and the median density of collagen fibers in the LWA was 60,000.00 (IQR $55000.00-75,000.00) / \mathrm{mm}^{2}$. Thus, when compared to the LWA, the density of collagen fibers in the HWA was significantly lower $(P=0.0177)$. Taken together, our data showed that the collagen fibers in the HWA were better arranged than the collagen fibers in the LWA.

\section{Discussion}

There are differences in macroscopic mechanical properties between the LWA and the HWA of cartilage [11-15]. Chondrocytes are inductive to mechanical effects at the microscopic level, therefore, it is important to know the microscopic mechanical properties and their relationship to macroscopic mechanical properties. Our findings showed that cartilage compressive modulus between HWA and LWA at the micro level was consistent with the trend observed at the macro level, while no significant differences in compressive modulus, diameter and structure of collagen fibers were found at the nano level between two areas. This information is critical to investigate the mechanical properties of cartilage on different load bearing areas. Furthermore, such information is crucial for the development of engineering technology of cartilage tissue. Insight into the micro and nano level of cartilage may provide novel insight for the development of cartilage replacement materials, and may serve as a reference for future research on the micro mechanical properties of osteoarthritis.

The low FI value as determined by our histological studies and the fact that no statistical differences were
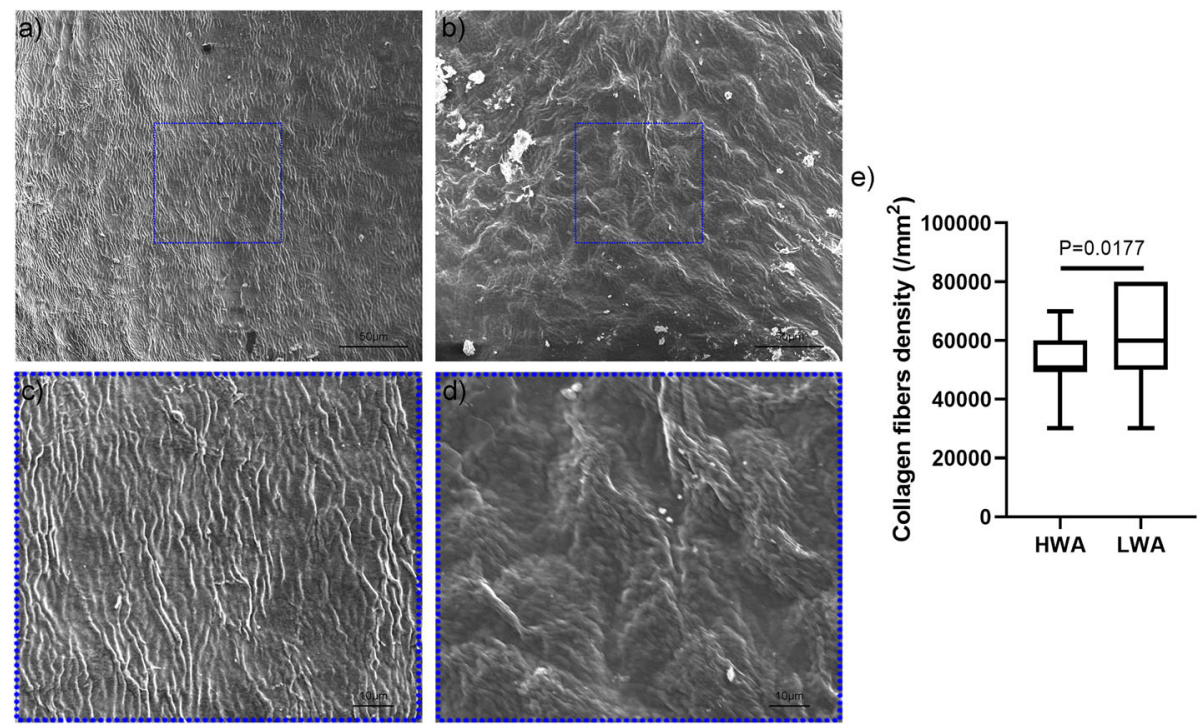

Fig. 5 Representative scanning electron microscope (SEM) micro-scale image of the cartilage morphology of the two areas. (a) High-weightbearing area (HWA) at a magnification of $1000 \times$. (b) Low-weight-bearing area (LWA) at a magnification of $1000 \times$. (c) HWA at a magnification of $3000 \times$. (d) LWA at a magnification of $3000 \times$. (e) The collagen fiber density of the two areas $(n=6$ per area) 
observed between the two groups indicated that we successfully established normal cartilage of the two areas [22]. The extracellular matrix of cartilage is mainly composed of collagen, proteoglycans, and water. The Safranin-O index was significantly higher in the HWA, thereby indicating that the HWA contained a higher proteoglycan content. These findings were consistent with the data presented in previous studies, in which was reported that a compressive modulus strongly and positively correlated with proteoglycan content $[29,30]$.

Regarding the macroscopic view of the influence of different weight-bearing level on the elastic modulus of articular cartilage, Shaw et al. reported the effect of different weight-bearing level on the tensile modulus of the knee joint of healthy individuals [11]. Their results demonstrated that the tensile modulus of the LWA was higher when compared to that of the HWA. However, Swann et al. reported that the level of cartilage stiffness was higher in the HWA in the knee and ankle joints [15]. Kempson et al. reported that the stiffest cartilage was located in a band, which extended from the superior surface of the femoral head, up to the anterior and posterior aspects, which indicated that the HWA had a higher modulus [14]. Furthermore, Athanasiou et al. reported that the superior portion of the femoral head cartilage has a higher modulus when compared to the inferior portion of the femoral head cartilage [12]. Karchner et al. reported a higher modulus in the HWA of the stifle joint [13]. The results mentioned above are similar to our macro-level results except for Shaw. The differences between Shaw and others may be attributed to the following, firstly, in macro mechanics, the experimental methods are different. The results from Shaw were measured by tensile testing using isometric tensile apparatus, whereas the results from others were measured by compression testing using indentation machines. Secondly, in previously published studies, the specimens were processed by freezing and thawing before being measured, and the freeze-thaw process may affect the mechanical properties of the specimen and introduce changes [31]. The number of freeze-thaw cycles should be kept to a minimum. Our study was the first to reveal differences in mechanical properties of different weight-bearing areas of normal femoral head cartilage at the micro and nano level, which complements the macro mechanical properties. Our findings showed that HWA cartilage maintained a high elastic modulus at the micro level. The elastic modulus of a single collagen fiber was measured at the nanometer level, and no significant differences were observed in the elastic modulus of collagen fibers between the two weightbearing areas. The weight-bearing level did not affect the single collagen fiber at the nanometer level, which suggested that the mechanical properties of cartilage at the macro- and micro-scale were affected by multiple factors, not just the stiffness of the collagen fiber [26].

The arrangement of collagen fibers mainly forms the elastic scaffold of the cartilage, and water absorption of the proteoglycans results in local elastic tension and osmotic tension [20]. The collagen cross-linking mechanism, proteoglycans, and water influenced by a different weight-bearing level caused significant differences in the elastic modulus at the macro- and micro-scale [19, 20, 32]. At the micro level, the distribution of collagen fibers in the HWA was more uniform when compared to that in the LWA. In addition, observation of the morphology of cartilage at the nano-scale level, including crosslinking mode and diameter of collagen fibers, revealed no significant differences between the two areas. This may be related to the daily weight-bearing activity in the HWA, which may result in a more organized collagen fiber arrangement and reconstruction to provide sufficient bearing capacity [19, 20,33]. This reconstruction was only reflected in the cartilage structure at the micro level, whereas the load has not yet affected the structure of collagen fibers at the nano-scale.

The present study has a number of inevitable limitations. Several differences were observed between in vitro-treated samples and direct in vivo detection. Because specimens for the unconfined compression test, SEM, and AFM need preparation, direct in vivo mechanical property measurements are impossible. In this study, we aimed for proper selection of animal specimens. Differences in walking patterns between animals and humans result in different cartilage stress conditions between the two species. However, the animal cartilage model has many advantages. Firstly, it is easier to control the integrity of porcine cartilage. As the human body grows, degeneration of the cartilage tissue occurs with age. The quality of porcine cartilage can be controlled by selecting young and healthy pigs without apparent degeneration, which are then used for experimental studies. Secondly, due to the similarities in cartilage structure and the vascular system between porcine and humans, porcine tissue has been widely used as a mechanical model for research [19, 32, 34, 35]. Because of the slight differences between human and porcine tissue, the porcine femoral head was used for investigating cartilage stress, thus the experiments closely resemble the human condition [36].

\section{Conclusions}

The results confirmed that a higher proteoglycan content in the HWA correlated with a higher micro compressive modulus in the HWA. Data at the micro-scale level was consistent with the trend observed by the macro compressive modulus. Moreover, the micro structure of cartilage may influence the macro- and micro- 
scale elastic modulus. However, weight-bearing was not associated with single collagen fibers at the nano level, and no significant differences were observed in the compressive modulus, structure, and diameter of the collagen fibers in the two areas. The above findings may provide novel insight for the development of cartilage replacement materials, studies on micromechanical properties of pathologically degenerated cartilage, among others.

\section{Abbreviations \\ HWA: High-weight-bearing area; LWA: Low-weight-bearing area; Fl: Fibrillation index; AFM: Atomic force microscope; SEM: Scanning electron microscope}

\section{Acknowledgements}

Not applicable.

\section{Authors' contributions}

ZPL contributed to the study design. JBG contributed to the SEM scanning and manuscript development. TL contributed to AFM biomechanical testing and data collection. YJC contributed to the data analysis. HLY was an advisor. All authors have read and approved the final manuscript.

\section{Funding}

This project is funded by the Basic Research Program of Jiangsu Province (BK20180196), National Natural Science Foundation of China (31570943), Innovation and Entrepreneurship Program of Jiangsu Province, and the Priority Academic Program Development of Jiangsu Higher Education Institutions, Natural Science Foundation of the Jiangsu Higher Education Institutions of China (18KJB180024), Jiangsu Planned Projects for Postdoctoral Research Funds (1601051C). The funding body did not influence the design of the study, the collection, analysis, or interpretation of the data, or the writing of the manuscript.

\section{Availability of data and materials}

The datasets used and/or analyzed during the current study are available from the corresponding author on reasonable request.

\section{Ethics approval and consent to participate}

All animal experiments were strictly performed under the guidelines of the Chinese Council for Animal Care, approved by the Animal Care Committee of the Laboratory Animal at School of Medicine, SooChow University.

\section{Consent for publication}

Not applicable.

\section{Competing interests}

The authors declare that they have no competing interests.

\section{Author details}

'Department of Orthopaedics, the First Affiliated Hospital of Soochow University, Suzhou, Jiangsu 215006, People's Republic of China. ${ }^{2}$ Department of Orthopaedics, Orthopaedic Institute, the First Affiliated Hospital, Soochow University, Suzhou, Jiangsu 215006, People's Republic of China.

Received: 26 January 2020 Accepted: 29 June 2020

Published online: 02 July 2020

\section{References}

1. Rydell N. Biomechanics of the hip-joint. Clin Orthop Relat Res. 1973;92:6-15.

2. Little CJ, Bawolin NK, Chen X. Mechanical properties of natural cartilage and tissue-engineered constructs. Tissue Eng Part B Rev. 2011;17(4):213-27.

3. Guilak F, Fermor B, Keefe FJ, Kraus VB, Olson SA, Pisetsky DS, et al. The role of biomechanics and inflammation in cartilage injury and repair. Clin Orthop Relat Res. 2004:423:17-26.

4. Li J, Wang Q, Jin Z, Williams S, Fisher J, Wilcox RK. Experimental validation of a new biphasic model of the contact mechanics of the porcine hip. Proc Inst Mech Eng H. 2014;228(6):547-55.
5. Pawaskar SS, Grosland NM, Ingham E, Fisher J, Jin Z. Hemiarthroplasty of hip joint: an experimental validation using porcine acetabulum. J Biomech. 2011;44(8):1536-42.

6. Toyoda E, Sato M, Takahashi T, Maehara M, Okada E, Wasai S, et al. Transcriptomic and proteomic analyses reveal the potential mode of action of chondrocyte sheets in hyaline cartilage regeneration. Int J Mol Sci. 2019; 21(1):149.

7. Won Y, Lee GS, Kim SB, Kim SJ, Yang KH. Osteochondral autograft from the Ipsilateral femoral head by surgical dislocation for treatment of femoral head fracture dislocation: a case report. Yonsei Med J. 2016;57(6):1527-30.

8. Nam D, Shindle MK, Buly RL, Kelly BT, Lorich DG. Traumatic osteochondral injury of the femoral head treated by mosaicplasty: a report of two cases. HSS J. 2010;6(2):228-34.

9. Ahsan T, Sah RL. Biomechanics of integrative cartilage repair. Osteoarthr Cartil. 1999:7(1):29-40

10. Guilak F, Butler DL, Goldstein SA. Functional tissue engineering: the role of biomechanics in articular cartilage repair. Clin Orthop Relat Res. 2001; 391 (Suppl):S295-305

11. Akizuki S, Mow VC, Muller F, Pita JC, Howell DS, Manicourt DH. Tensile properties of human knee joint cartilage: I. influence of ionic conditions, weight bearing, and fibrillation on the tensile modulus. J Orthop Res. 1986; 4(4):379-92.

12. Athanasiou KA, Agarwal A, Muffoletto A, Dzida FJ, Constantinides G, Clem M. Biomechanical properties of hip cartilage in experimental animal models. Clin Orthop Relat Res. 1995;316:254-66.

13. Karchner JP, Yousefi F, Bitman SR, Darvish K, Pleshko N. Non-destructive spectroscopic assessment of high and low weight bearing articular cartilage correlates with mechanical properties. Cartilage. 2019;10(4):480-90.

14. Kempson GE, Spivey CJ, Swanson SA, Freeman MA. Patterns of cartilage stiffness on normal and degenerate human femoral heads. J Biomech. 1971; 4(6):597-609.

15. Swann AC, Seedhom BB. The stiffness of normal articular cartilage and the predominant acting stress levels: implications for the aetiology of osteoarthrosis. Br J Rheumatol. 1993;32(1):16-25.

16. Discher D, Dong C, Fredberg JJ, Guilak F, Ingber D, Janmey P, et al. Biomechanics: cell research and applications for the next decade. Ann Biomed Eng. 2009;37(5):847-59.

17. Muir $\mathrm{H}$. The chondrocyte, architect of cartilage. Biomechanics, structure, function and molecular biology of cartilage matrix macromolecules. BioEssays. 1995:17(12):1039-48.

18. Pizzo AM, Kokini K, Vaughn LC, Waisner BZ, Voytik-Harbin SL. Extracellular matrix (ECM) microstructural composition regulates local cell-ECM biomechanics and fundamental fibroblast behavior: a multidimensional perspective. J Appl Physiol (1985). 2005;98(5):1909-21.

19. Sanchez-Adams J, Wilusz RE, Guilak F. Atomic force microscopy reveals regional variations in the micromechanical properties of the pericellular and extracellular matrices of the meniscus. J Orthop Res. 2013;31(8):1218-25.

20. Franz T, Hasler EM, Hagg R, Weiler C, Jakob RP, Mainil-Varlet P. In situ compressive stiffness, biochemical composition, and structural integrity of articular cartilage of the human knee joint. Osteoarthr Cartil. 2001;9(6):58292.

21. Antons J, Marascio MGM, Nohava J, Martin R, Applegate LA, Bourban PE, et al. Zone-dependent mechanical properties of human articular cartilage obtained by indentation measurements. J Mater Sci Mater Med. 2018;29(5): 57.

22. Pastoureau P, Leduc S, Chomel A, De Ceuninck F. Quantitative assessment of articular cartilage and subchondral bone histology in the meniscectomized Guinea pig model of osteoarthritis. Osteoarthr Cartil. 2003; 11(6):412-23.

23. Raya JG, Arnoldi AP, Weber DL, Filidoro L, Dietrich O, Adam-Neumair S, et al. Ultra-high field diffusion tensor imaging of articular cartilage correlated with histology and scanning electron microscopy. MAGMA. 2011;24(4):247-58.

24. Korhonen RK, Laasanen MS, Toyras J, Rieppo J, Hirvonen J, Helminen HJ, et al. Comparison of the equilibrium response of articular cartilage in unconfined compression, confined compression and indentation. J Biomech. 2002;35(7):903-9.

25. Zopf DA, Flanagan CL, Nasser HB, Mitsak AG, Huq FS, Rajendran V, et al. Biomechanical evaluation of human and porcine auricular cartilage. Laryngoscope. 2015;125(8):E262-8. 
26. Liang T, Che YJ, Chen X, Li HT, Yang HL, Luo ZP. Nano and micro biomechanical alterations of annulus fibrosus after in situ immobilization revealed by atomic force microscopy. J Orthop Res. 2019;37(1):232-8.

27. Nakano $\mathrm{T}$. $\mathrm{NaOH}$ cell maceration/scanning electron microscopic studies on the connective tissue core in the incisive portion of the mouse hard palate. Auris Nasus Larynx. 1995;22(1):29-37.

28. Reginato Gde S, Bolina Cde S, Watanabe IS, Ciena AP. Three-dimensiona aspects of the lingual papillae and their connective tissue cores in the tongue of rats: a scanning electron microscope study. ScientificWorldJournal. 2014;2014:841879.

29. Kiviranta P, Rieppo J, Korhonen RK, Julkunen P, Toyras J, Jurvelin JS. Collagen network primarily controls Poisson's ratio of bovine articular cartilage in compression. J Orthop Res. 2006;24(4):690-9.

30. Lakin BA, Ellis DJ, Shelofsky JS, Freedman JD, Grinstaff MW, Snyder BD. Contrast-enhanced CT facilitates rapid, non-destructive assessment of cartilage and bone properties of the human metacarpal. Osteoarthr Cartil. 2015;23(12):2158-66.

31. Quirk NP, Lopez De Padilla C, De La Vega RE, Coenen MJ, Tovar A, Evans $\mathrm{CH}$, et al. Effects of freeze-thaw on the biomechanical and structural properties of the rat Achilles tendon. J Biomech. 2018;81:52-7.

32. Imer R, Akiyama T, de Rooij NF, Stolz M, Aebi U, Friederich NF, et al. The measurement of biomechanical properties of porcine articular cartilage using atomic force microscopy. Arch Histol Cytol. 2009;72(4-5):251-9.

33. Changoor A, Nelea M, Methot S, Tran-Khanh N, Chevrier A, Restrepo A, et al, Structural characteristics of the collagen network in human normal, degraded and repair articular cartilages observed in polarized light and scanning electron microscopies. Osteoarthr Cartil. 2011;19(12):1458-68.

34. Cone SG, Warren PB, Fisher MB. Rise of the pigs: utilization of the porcine model to study musculoskeletal biomechanics and tissue engineering during skeletal growth. Tissue Eng Part C Methods. 2017;23(11):763-80.

35. Franke O, Goken M, Meyers MA, Durst K, Hodge AM. Dynamic nanoindentation of articular porcine cartilage. Mat Sci Eng C-Mater. 2011; 31(4):789-95.

36. Taylor SD, Tsiridis E, Ingham E, Jin Z, Fisher J, Williams S. Comparison of human and animal femoral head chondral properties and geometries. Proc Inst Mech Eng H. 2012;226(1):55-62.

\section{Publisher's Note}

Springer Nature remains neutral with regard to jurisdictional claims in published maps and institutional affiliations.

Ready to submit your research? Choose BMC and benefit from:

- fast, convenient online submission

- thorough peer review by experienced researchers in your field

- rapid publication on acceptance

- support for research data, including large and complex data types

- gold Open Access which fosters wider collaboration and increased citations

- maximum visibility for your research: over $100 \mathrm{M}$ website views per year

At BMC, research is always in progress.

Learn more biomedcentral.com/submissions 